\title{
Travel to the Center of Atomic Nuclei
}

\author{
Gianni Donati \\ Associazione Italiana di Ingegneria, Chimica, Italy \\ Email: gia.donati@tiscali.it
}

How to cite this paper: Donati, G. (2019) Travel to the Center of Atomic Nuclei. Journal of Applied Mathematics and Physics, 7, 2352-2372.

https://doi.org/10.4236/jamp.2019.710160

Received: September 11, 2019

Accepted: October 19, 2019

Published: October 22, 2019

Copyright (c) 2019 by author(s) and Scientific Research Publishing Inc. This work is licensed under the Creative Commons Attribution International License (CC BY 4.0).

http://creativecommons.org/licenses/by/4.0/

\begin{abstract}
Nuclei, with their internal proton-neutron transformations, electron and neutrino emission, are the ultimate motor of the universe both at the atomic and cosmological scale. The flux of neutrino governs the motion of the Earth around the Sun, the electronic orbital, the physical-chemical properties of substances, while the proton-neutron transformations are responsible for the nuclear bond and nuclear stability. The mechanical and chemical world is well known and understood while the structure of the nucleus and the nature of the nuclear bond are still a mystery due to the lack of experimental techniques at the subatomic scale. The recent consciousness of nuclei global role at the macroscale forces additional investigations. In our travel, we use, as a guide, the available mass defect data, propose a new approach to reconcile unexplained binding energies of lighter nuclides and give additional rules to build stepwise all known isotopes. While the rules appear to be sound, the picture that comes out is however only a first rough tentative of radiography of the internal structure of the nuclei of existing atoms.
\end{abstract}

\section{Keywords}

Physics, Nuclear Bond, Particle Physics, Gravity, Grand Unified Theory

\section{Introduction}

Understanding the nature of the forces that hold nuclei together is one of the two main challenges of physics, the other being the gravitational interaction.

In a previous paper dated 2004 [1], we anticipated a new vision of the strong nuclear interaction, as the other face of the same coin holding together the strong and the week interaction.

Gravity has been the obsession of the last century with the work of Einstein dominating the scene, but now that the new ideas on gravity begin to get ground, we are forced to go deeper inside the nature of the strong nuclear interaction and the structure of atomic nuclei. 
A lot of precise and accurate data are available on stable and unstable nuclides for the computation of the nuclear interaction energy that is related to the mass defect, that is to the difference between the measured mass and the sum of the $Z$ hydrogen ${ }^{1} \mathrm{H}$ (or protons $\boldsymbol{p}$ plus electron $\boldsymbol{e}$ ) masses and $N$ neutron $\boldsymbol{n}$ masses that compose the nucleus of mass number $A=Z+N$ and mass $M$.

$$
\Delta m=[Z m(1 H)+N m n-M]=[Z(m p+m e)+N m n-M]
$$

Masses are usually given in amu (atomic mass unit) $=\mathrm{Da}$ (Dalton), that is $1 / 12$ the mass of ${ }^{12} \mathrm{C}$, that conventionally has a mass of $12 \mathrm{amu}$ and are usually transformed in energy units: amu/Mev equals to 931.5.

The neutron is unstable and decades to a hydrogen atom with a half life of 15 minutes while an isolated hydrogen atom appears to leave forever.

A neutron has a slightly greater mass than a proton by $0.78246 \mathrm{Mev}$ and even greater than the mass of hydrogen by $0.27144875 \mathrm{Mev}$, with the electron weighting $0.51101125 \mathrm{Mev}$.

There are many hypotheses for this $0.27144875 \mathrm{Mev}$ mass difference, but a universally accepted hypothesis does not exist, even if, in the neutron hydrogen transformation, it may remain hidden in the hydrogen atom in some form of unknown energy, other than mass.

The recent proposal by Borsanyi et al. [2] that this tiny mass splitting is the result of a subtle cancellation between electromagnetic and quark mass, goes in this direction.

An implicit assumption in Equation (1) for the mass defect representing the nuclear binding energy, is that this unknown energy remains the same, in the transformation, for the proton-electron pairs in the nucleus.

As a matter of facts, Equation (1) is not a mass/energy balance and sometimes the mass defect is computed using the neutron weight alone and thus incorporating this unknown energy:

$$
\Delta m=[A m n-M]
$$

In addition the nucleus is thought to include other forms of energy like the repulsion energy due to the proton charges that should oppose the strong nuclear interaction.

These are considered in the liquid drop model [1] as an electrostatic Coulomb potential $C$ proportional to the square of the electron charge and to the inverse of nucleus size.

This contribution, if supposed due to an additional mass loss, should be added to $\Delta m$, resulting in an apparent rapid increase of binding energy with the atomic number $Z$.

$$
\Delta m=[Z m(1 H)+N m n-M]+C
$$

In the already mentioned paper [1] we have made a fitting of the liquid drop model over all available nuclides using the following equation for Coulomb energy (Mev); 


$$
C=4 \times 0.146 Z(Z-1) / A^{\frac{1}{3}}
$$

The nucleus is not however static and the way this contribution should be considered is still under discussion, given that there are additional problems to be solved.

It is indeed unlike that the nucleus should be synthesized from its elementary constituents, given that the Sun is able to produce, by fusion, only the first three elements of the periodic table, the remaining eventually been generated far away in extreme condition in other stars at the end of their lifecycle.

The big puzzle in nuclear physics is however the relative binding energies of the small nuclides, particularly deuterium, tritium and alpha particle.

The Deuteron, the Hydrogen 2 nuclide, has binding energy of about 2.225 $\mathrm{MeV}$ (Equation (1)).

The tritium, the Hydrogen 3 nuclide, should have binding energy of about $8.48 \mathrm{MeV}$.

This is a very large increase, but the jump to alpha particle, Helium 4 nuclide, is even larger.

The binding energy of the alpha particle might be about $28.29 \mathrm{MeV}$.

Note, for additional comparison, that the Helium 3 nuclide has binding energy of about $7.72 \mathrm{MeV}$.

The Helium 3 nuclide differs from the tritium in that it has two protons and one neutron rather than one proton and two neutrons.

The liquid drop model does not fit these anomalies but the fitting improves with the atomic number to Neon and the model explains more than 99.9 of the binding energy of heavier Nuclides.

The nature of the nuclear bond remains obscure and to find some light we begin a trip starting from Deuterium and stopping to Neon, not to bore booth the author and the reader.

In Section 2 we introduce our view of the nuclear bond and in Section 3 we show how this view can solve the problem of the excessive binding energies of Tritium and Helium isotopes.

Section 4 presents a geometric extrapolation to heavier nuclides of Helium tetrahedron shape as suggested by the most recent investigations.

Sections 5 and 6 show the rules and demonstrate with examples how to proceed along the path of heavier nuclides

\section{The Nature of the Nuclear Bond}

Our travel inside the structure of the nucleus should be controlled by the mass defect, that represents the only numerical value available for the evaluation of the nuclear binding energy, in spite of the mentioned problems.

We know however that the nucleus is far from being a static mix of nucleons generating a static electron's orbital.

We were inspired by the theory of $\beta$ decay by E. Fermi [3] to derive the new vision of gravitation and now we are comforted to use it as a starting point to 
define the rules to be followed in our trip inside nuclei.

Protons $p$ and neutrons $n$ are subject to dynamic transformations:

$\beta^{-}$emission

$$
n \frac{k 1}{\leftrightarrow} p+\beta^{-}+v
$$

$\beta^{+}$emission

$$
p \frac{k 2}{\leftrightarrow} n+\beta^{+}+v
$$

$$
\text { Orbital electron capture } \quad p+\beta \frac{k 3}{\leftrightarrow} n+v
$$

and the electron-positron $\left(\beta^{-}-\beta^{+}\right)$annihilation reaction with the production of two $\gamma$ photons having energy of $0.511 \mathrm{Mev}$ each, equal to the rest energy of an electron.

$$
\beta^{-}+\beta^{+}=2 \gamma
$$

E. Fermi used these equations to describe the dynamics of $\beta$ emission in radioactive nuclides and that was the first time that the existence of neutrino was justified.

We used this representation even for non radioactive nuclides where electrons are not emitted, but captured back and neutrino is only the cooled version of electromagnetic radiation.

The emission of radiation till its final neutrino form represents the specific heat and the internal energy of matter, the neutrino emission determines the gravitational forces in the universe, the electron energy levels define the chemical behaviour of elements and the underlying neutron-proton transformations the dynamic nature of the nuclear bond, annihilation reaction included.

We stated [1] [4] that this mechanism is valid for all nuclides and this is the way how nuclei control, at the same time, the electron's shells and the shape of the universe.

The constants k1, k2, k3 of reactions (5) can be computed on the basis of the n-p distribution in existing nuclides, are universal constants and allow the computation of the neutrino flux Fo (neutrino per gram of matter and per second) that is surprisingly almost constant for all nuclides.

Fo is related to the Gauss constant $G$ in Newton Gravitational Law by the following equation:

$$
G=F o \mu r_{n}^{2} c / 4 m o
$$

where $F o=6.668 \mathrm{E}+20 \mathrm{v} /(\mathrm{g} \cdot \mathrm{s})$ is the neutrino flux, $\mu=1.55277 \mathrm{E}-36 \mathrm{~g}$ is the neutrino mass, $c$ is the speed of light and mo, $\boldsymbol{r}_{\boldsymbol{n}}$ are the nucleon mass (g) and radius $(\mathrm{cm})$.

The neutrino/graviton emission and the proton-neutron distribution in all known nuclide allowed us to solve some remaining question marks about the motion of Galaxy [5], the delay in the Earth revolution and the change of its distance from the Sun [6] and even to define a new hypothesis on Earthquakes generation [7].

In addition an analogy to the electromagnetic field has been devised [8], the effect on the specific energy of matter [9] and on the velocity of light [10] have been demonstrated as reported in a recent review [11]. 
In [1] we anticipated that there is no force holding nucleons together but just the ability of proton and neutron to share the matter that has been lost during the formation of nuclei. This happens on product nuclei of $\beta$ decay with the release of energy and the formation of new bonds.

We suggested, contrarily to the common opinion, that only $\mathrm{p}-\mathrm{n}$ bonds are possible and that the energy involved is equivalent to two annihilation reactions that is a $2.044 \mathrm{Mev}$, “double nuclear bond" formation, in which neutron and proton share part of themselves like conjoined twins.

One neutron or proton can be multiply conjoined with protons or neutrons and nucleons can change partner during $n-p$ transformations.

In Table 1 we report the physical properties of all known nuclides to Neon and compute the binding energy and the number of bonds following Equations (1), (2) and (3).

Equation (1) appears to be the most regular, while Equation (2) takes into account the n-H1 difference, overestimates lighter nuclide and adds a positive correction for the heavier ones.

Equation (3) adds Coulomb energy, that soars with the square of the atomic number $\mathrm{Z}$, is almost equivalent to (1) for lighter nuclides but diverges from (2) for the heavier ones.

Table 1 provides also an idea of the incertitude in estimating, from mass defect, the number of $n-p$ bonds under the $2.044 \mathrm{Mev}$ hypotheses.

\section{Lighter Nuclides}

The surprisingly high number of bonds of lighter nuclides can be related to the dynamic behavior of the nucleus under transformations (5).

In Figure 1 the two states of Deuterium ${ }^{2} \mathrm{H}$ with proton 1 bond to neutron 2 and neutron 1 to proton 2 resulting in one double bond while for Tritium and Helium 3 we have three possible transformations.

For Tritium ${ }^{3} \mathrm{H}$, in the three possible combinations, we obtained 4 non repeated bonds.

For ${ }^{3} \mathrm{He}$ we get a similar result that is surprisingly in line with the experimental value of Table 1 and explains the anomalies of these light nuclides compared with the heavy ones.

Coming to ${ }^{4} \mathrm{He}$, the puzzle becomes clearer, as shown in Figure 2, where the number of dynamic states of the nucleus is six and the number of dynamic bond is 14 , again in line with the experimental values reported in Table 1 under the 2.044 Mev hypotheses. All these bonds are at the same time statistically present.

We derive in addition the idea that the tetrahedron shape of ${ }^{4} \mathrm{He}$ should dominate the scene and all resonant shapes are present during the nucleons dynamic transformations.

We wonder if the heavier nuclides follow the same rule and for continuing the representation we need to imagine a geometric representation for the growth of atomic nucleus. 
Table 1. Nuclear Bonds from Experimental Data.

\begin{tabular}{|c|c|c|c|c|c|c|c|c|c|c|c|c|c|c|c|}
\hline & $\mathrm{Z}$ & A & $\mathrm{N}$ & halflife & Decay & Decay & $\begin{array}{l}\text { Decay } \\
\text { energy }\end{array}$ & MASS & Packing & m (1) & $\square \mathrm{m}(2)$ & $\square \mathrm{m}$ (3) & N.bond & N.bond & N.bond \\
\hline Nuclide & & & & s & mode & Mev & Mev & $\begin{array}{c}\mathrm{AMU}= \\
\mathrm{Da}\end{array}$ & & Mev & Mev & Mev & (1) & (2) & (3) \\
\hline $\mathrm{n} 1-\mathrm{H} 1$ & & & & & & & & 0.00084 & 931.5 & 0.7825 & & & & & \\
\hline e & & & & & & & & 0.00055 & & 0.511 & & & & & \\
\hline $\mathrm{N} 1-\mathrm{H} 1-\mathrm{e}$ & & & & & & & & 0.00029 & & 0.2714 & & & & & \\
\hline $\mathrm{n} 1$ & 0 & 1 & 1 & -720 & b- & 0.783 & 0.7825 & 1.0087 & 86.6 & 0 & 0 & 0 & & & \\
\hline $\mathrm{H} 1$ & 1 & 1 & 0 & & & & & 1.0078 & 78.2 & 0 & 0.782 & 0 & 0 & & \\
\hline $\mathrm{H} 2$ & 1 & 2 & 1 & & & & & 2.0140 & 70.0 & 2.32 & 3.10 & 2.32 & 1.1 & 1.5 & 1.1 \\
\hline $\mathrm{H} 3$ & 1 & 3 & 2 & $-3.9 \mathrm{E}+08$ & $b-$ & 0.019 & 0.0186 & 3.0161 & 53.5 & 8.48 & 9.26 & 8.48 & 4.1 & 4.5 & 4.1 \\
\hline He3 & 2 & 3 & 1 & & & & & 3.0160 & 53.4 & 7.72 & 9.28 & 8.53 & 3.8 & 4.5 & 4.2 \\
\hline $\mathrm{He} 4$ & 2 & 4 & 2 & & & & & 4.0026 & 6.5 & 28.30 & 29.86 & 29.03 & 13.8 & 14.6 & 14.2 \\
\hline He5 & 2 & 5 & 3 & & & & & 5.0122 & 24.4 & 27.43 & 28.99 & 28.11 & 13.4 & 14.2 & 13.8 \\
\hline He6 & 2 & 6 & 4 & -0.808 & b- & 3.51 & 3.5071 & 6.0189 & 31.5 & 29.27 & 30.84 & 29.91 & 14.3 & 15.1 & 14.6 \\
\hline $\mathrm{He} 8$ & 2 & 8 & 6 & -0.122 & $b-$ & 14 & 10.6517 & 8.0339 & 42.4 & 31.41 & 32.98 & 31.99 & 15.4 & 16.1 & 15.7 \\
\hline Li5 & 3 & 5 & 2 & & & & & 5.0125 & 25.1 & 26.33 & 28.68 & 28.38 & 12.9 & 14.0 & 13.9 \\
\hline Li6 & 3 & 6 & 3 & & & & & 6.0151 & 25.2 & 32.00 & 34.34 & 33.92 & 15.7 & 16.8 & 16.6 \\
\hline Li7 & 3 & 7 & 4 & & & & & 7.0160 & 22.9 & 39.25 & 41.59 & 41.08 & 19.2 & 20.3 & 20.1 \\
\hline $\mathrm{Li} 8$ & 3 & 8 & 5 & -0.844 & $\mathrm{~b}-, \mathrm{a}$ & 16.01 & 16.0050 & 8.0225 & 28.1 & 41.28 & 43.63 & 43.03 & 20.2 & 21.3 & 21.1 \\
\hline Li9 & 3 & 9 & 6 & -0.178 & $\mathrm{~b}-$ & 13.61 & 13.6064 & 9.0268 & 29.8 & 45.34 & 47.69 & 47.03 & 22.2 & 23.3 & 23.0 \\
\hline Be6 & 3 & 6 & 3 & & & & & 6.0197 & 32.9 & 27.71 & 30.05 & 29.64 & 13.6 & 14.7 & 14.5 \\
\hline Be7 & 4 & 7 & 3 & 4604256 & EC & 0.862 & 0.8616 & 7.0169 & 24.2 & 37.60 & 40.73 & 41.27 & 18.4 & 19.9 & 20.2 \\
\hline Be9 & 4 & 9 & 5 & & & & & 9.0122 & 13.5 & 58.17 & 61.30 & 61.53 & 28.5 & 30.0 & 30.1 \\
\hline Be10 & 4 & 10 & 6 & $-5 E+13$ & $b-$ & 0.556 & 0.5561 & 10.0135 & 13.5 & 64.98 & 68.11 & 68.23 & 31.8 & 33.3 & 33.4 \\
\hline Be11 & 4 & 11 & 7 & -13.8 & $\mathrm{~b}-, \mathrm{b}-\mathrm{a}$ & 11.48 & 11.5068 & 11.0217 & 19.7 & 65.48 & 68.61 & 68.63 & 32.0 & 33.6 & 33.6 \\
\hline B8 & 5 & 8 & 3 & 0.772 & $\mathrm{~b}+, 2 \mathrm{a}$ & & 11.0000 & 8.0246 & 30.8 & 37.74 & 41.65 & 43.58 & 18.5 & 20.4 & 21.3 \\
\hline B10 & 5 & 10 & 5 & & & & & 10.0129 & 12.9 & 64.75 & 68.66 & 70.17 & 31.7 & 33.6 & 34.3 \\
\hline B11 & 5 & 11 & 6 & & & & & 11.0093 & 8.5 & 76.21 & 80.12 & 81.46 & 37.3 & 39.2 & 39.9 \\
\hline B12 & 5 & 12 & 7 & -0.0202 & $b-$ & 13.37 & 13.3689 & 12.0144 & 12.0 & 79.58 & 83.49 & 84.68 & 38.9 & 40.8 & 41.4 \\
\hline B13 & 5 & 13 & 8 & -0.0173 & $b-$ & 13.44 & 13.4369 & 13.0178 & 13.7 & 84.45 & 88.37 & 89.42 & 41.3 & 43.2 & 43.7 \\
\hline C9 & 6 & 9 & 3 & 0.127 & $\mathrm{~b}+, \mathrm{P} 2 \mathrm{a}$ & 16.5 & 16.4970 & 9.0310 & 34.5 & 39.04 & 43.73 & 47.46 & 19.1 & 21.4 & 23.2 \\
\hline $\mathrm{C} 10$ & 6 & 10 & 4 & 19.3 & $\mathrm{~b}+$ & 3.65 & 3.7474 & 10.0170 & 17.0 & 60.22 & 64.92 & 68.35 & 29.5 & 31.8 & 33.4 \\
\hline $\mathrm{C} 11$ & 6 & 11 & 5 & 1218 & $\mathrm{~b}+, \mathrm{EC}$ & 1.982 & 1.9794 & 11.0114 & 10.4 & 73.44 & 78.14 & 81.32 & 35.9 & 38.2 & 39.8 \\
\hline $\mathrm{C} 12$ & 6 & 12 & 6 & & & & & 12.0000 & 0.0 & 92.16 & 96.86 & 99.82 & 45.1 & 47.4 & 48.8 \\
\hline $\mathrm{C} 13$ & 6 & 13 & 7 & & & & & 13.0034 & 2.6 & 97.11 & 101.80 & 104.56 & 47.5 & 49.8 & 51.2 \\
\hline $\mathrm{C} 14$ & 6 & 14 & 8 & $-1.8 \mathrm{E}+11$ & $\mathrm{~b}-$ & 0.156 & 0.1556 & 14.0032 & 2.3 & 105.29 & 109.98 & 112.56 & 51.5 & 53.8 & 55.1 \\
\hline
\end{tabular}




\section{Continued}

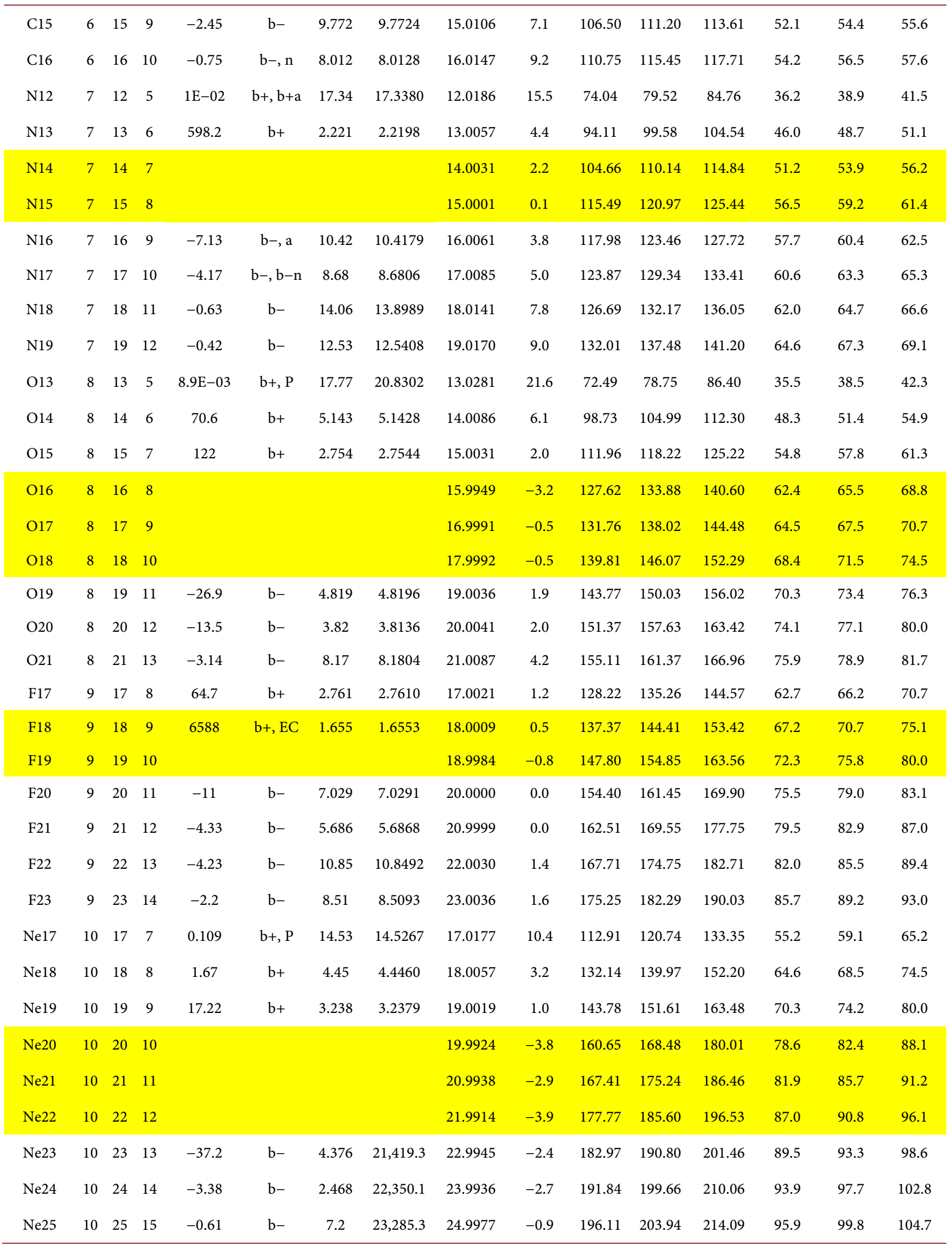




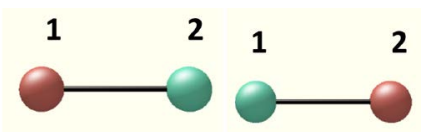

${ }^{2} \mathbf{H}$

121

$\begin{array}{lll}21 & 0 & >1\end{array}$
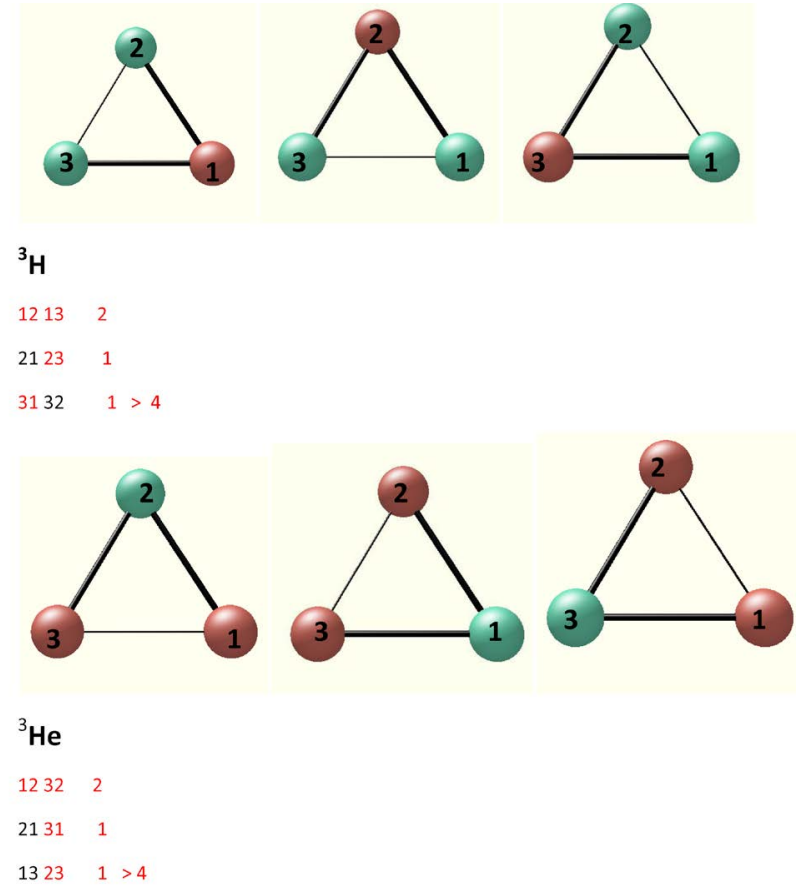

Figure 1. Number of dynamic bonds of ${ }^{2} \mathrm{H},{ }^{3} \mathrm{H}$ and ${ }^{3} \mathrm{He}$.
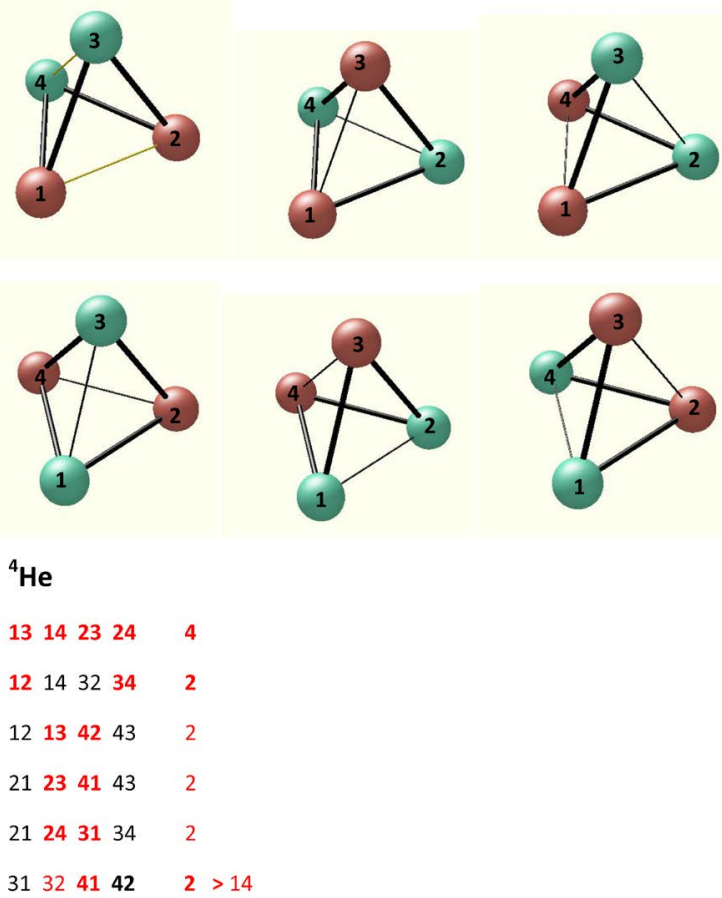

Figure 2. Number of dynamic bonds of ${ }^{4} \mathrm{He}$. 
The number of bonds computed with this simple geometric scheme fit very well the experimental data reported in Table 1, nothing to do with the complex calculation reported in the recent literature [12] [13].

Note that bond ij is equivalent to bond ji, black number are repeated bonds, red are non repeated ones and the count always starts from the smallest number red ball to avoid ambiguities with balls with numbers greater than 10 . The total number of bonds is the sum of nonrepeated bonds (red numbers). The figures represented in these and the following tables have been designed using Microsoft Ball \& Stich software where, for clarity, a stick length much greater than ball diameter has been used.

\section{The Geometric Representation of Heavier Nuclides}

Everybody agrees on the geometry of Helium isotopes and the recent literature is full of alpha clustering studies [14] [15] [16] [17] proposing that heavier nuclides should be built up as clusters of these elementary nuclides.

Alfa clustering in nuclei, at present is well studied and reasonably well accepted property of nucleus and a search for experimental evidence followed by complex calculation is under way.

On the other side Bihari G. [18] thinks that modeling atomic nucleus with a set of magnetic balls may be a sound shortcut, providing a macroscopic visualization of complex nuclear features.

In this paper we combine the advantage of booth, eliminating confusing computational complexities and recuperating the irrational of ball arrangement.

The tetrahedron shape of ${ }^{4} \mathrm{He}$ is maintained through the resonance of protons and neutrons operating in the nucleus center like an electrodynamics machine for shooting electrons and neutrino.

Adding neutrons or protons expands the tetrahedron with new bonds, but also introduces new constraints to neutrons-protons transformations.

Starting from ${ }^{4} \mathrm{He}$ (gold tetrahedron) we can easily design the four tetrahedron on each face by projection of the gold spheres through the opposite face to generate the silver ones (Figure 3).

This includes $\mathrm{Li}$ and $\mathrm{Be}$ with some of their isotopes and $\mathrm{He}$ isotopes. If we continue the projection of the gold spheres on the other side of the twelve silver faces we obtain the magenta tetrahedrons with only six new vertices This scheme includes B and C with some of their isotopes.

However we recognize that each of these six vertices can be obtained by two different projections, the allocation in space being slightly different, as shown in Figure 4(a) and Figure 4(b).

Continuing the projections we can expand the structure to include N, O, F and Ne.

We realize that a polytetrahedron structure is far from being a solid perfectly filling the space, as Aristotle believed, and we imagine this defect increasing with the atomic number with distortions and instabilities in the surface with the emission of alpha particles (Figure 5). 


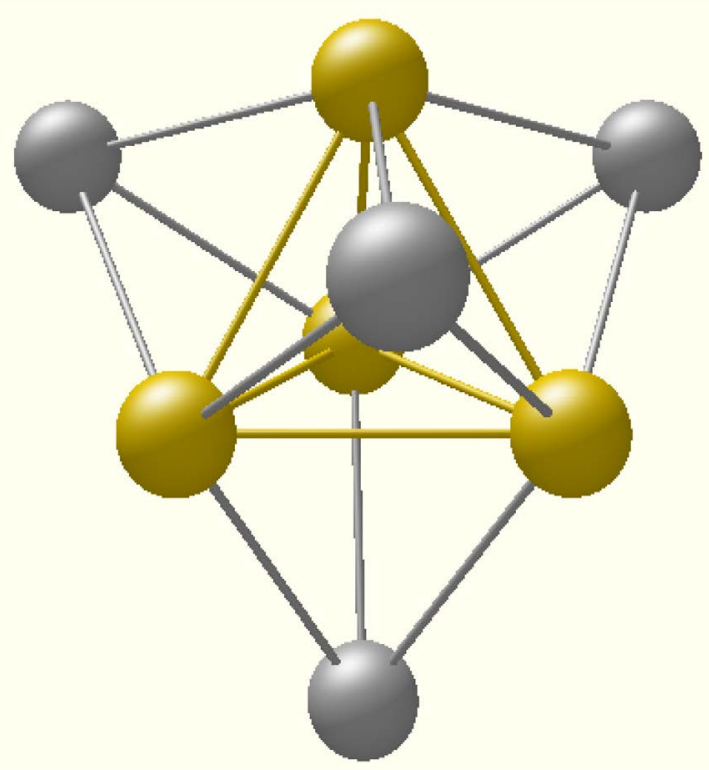

Figure 3. Adding four tetrahedrons to the core of ${ }^{4} \mathrm{He}$.

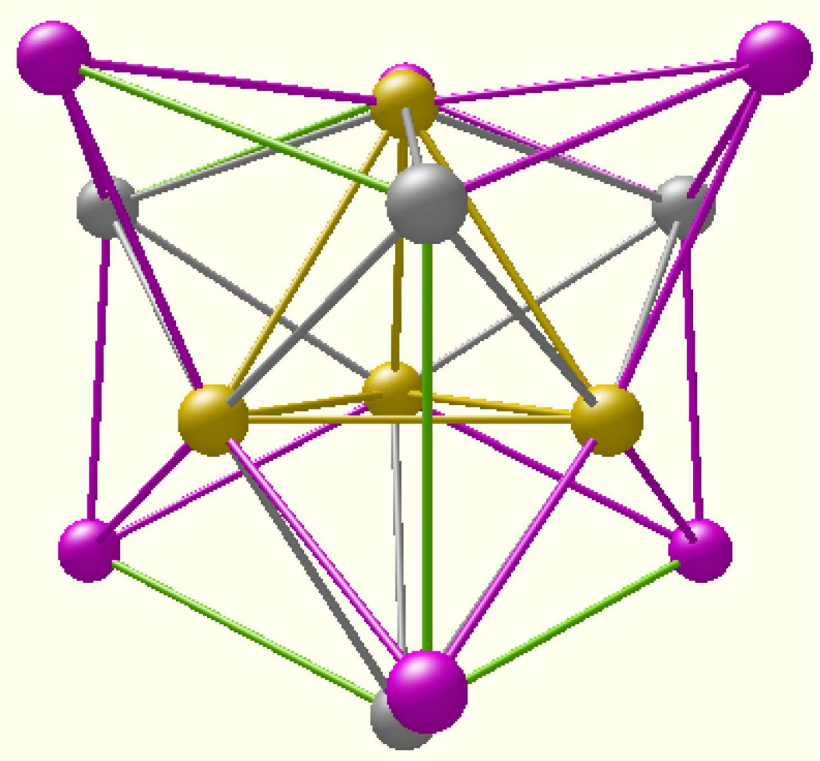

(a)

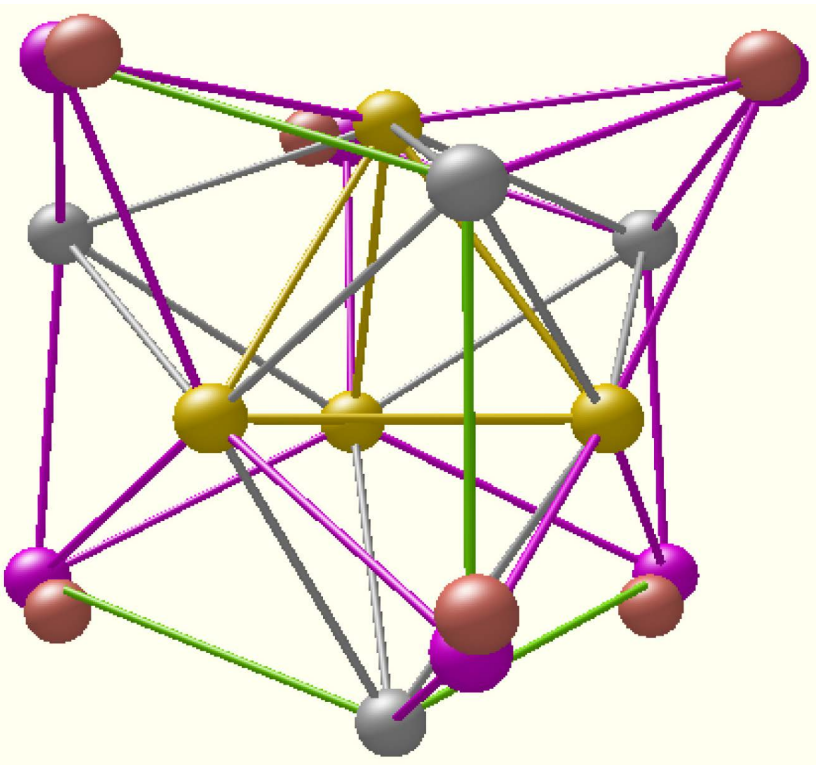

(b)

Figure 4. (a) Adding next six tetrahedrons to provide nucleons for B and C; (b) Adding next six tetrahedrons for nucleons $\mathrm{r} \mathrm{B}$ and $\mathrm{C}$ using different projections.

Even with 20 nucleons the stretching and relaxing of stick length are evidently introducing a possible geometric limit to the maximum atomic number alone.

The electrostatic repulsion in a dynamic system may not be a real obstacle for bond formation.

The exchange of the electric charge between proton and neutron is, in fact, the cause of the formation of the nuclear bond with the positive charge continuously changing location, and the electron participating to the game. 


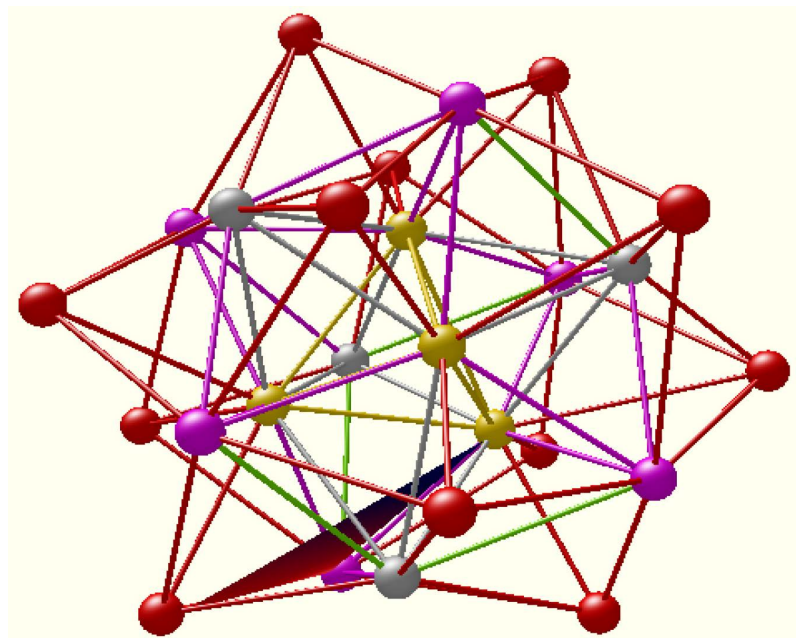

Figure 5. Adding nucleons to reach $\mathrm{Ne}$.

\section{Continuing the Travel with Lighter Nuclides}

The magic of tetrahedron geometry, even if geometrically imperfect, allows the building of all existing nuclides and even figure out new nuclides by addition of protons and neutrons.

We however have to realize that adding a new nucleon increases the degrees of freedoms but also introduces new constraints.

For stability reasons a tetrahedron should contain two neutrons and two protons and more generally each proton should be connected at least to two neutrons and vice versa.

Nucleons are fixed in the tetrahedron net and only for simpler nuclides some distortion is allowed, for example in ${ }^{5} \mathrm{He}$ only three stable variant are obtained and a forth is generated by a rotation of neutron 5 (Figure 6).

Adding the rotation we have 14 bonds against the 13.4 bonds computed with (1) the isotope ${ }^{5} \mathrm{Li}$ has a similar structure as reported in_Figure 7.

${ }^{5} \mathrm{He}$ is limited in the number of transformations and can rearrange rotating an appended neutron while ${ }^{5} \mathrm{Li}$ rotates the appended proton to reproduce a scheme similar to the preceding ones: this rotation may be required only for some light nuclides.

We have made the analysis of all stable nuclides of $\mathrm{Li}, \mathrm{Be}$ and $\mathrm{B}$ and we report, for example, in Figures 8-10 the computed number of dynamic bonds for the isotopes having higher relative abundance.

\section{The Path from $\mathrm{C}$ to $\mathrm{Ne}$}

Increasing the atomic number, the degrees of freedom are also increased and the complexity of the structure requires additional rules.

Structures having different number of bonds are possible as an alternative to a dominating series of structures having the same number of bonds.

Nuclei with less bond than the dominating one can evolve to a higher number of bonds, while those with a high number of bonds cannot reduce their 

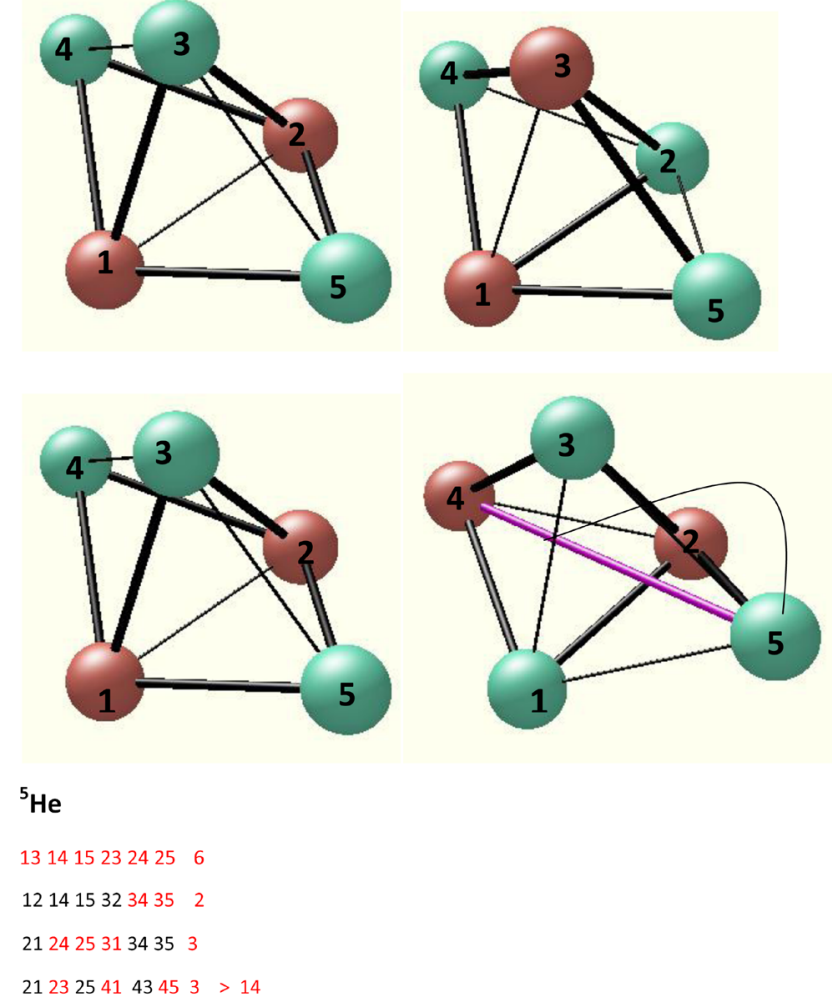

Figure 6. Number of dynamic bonds of ${ }^{5} \mathrm{He}$.
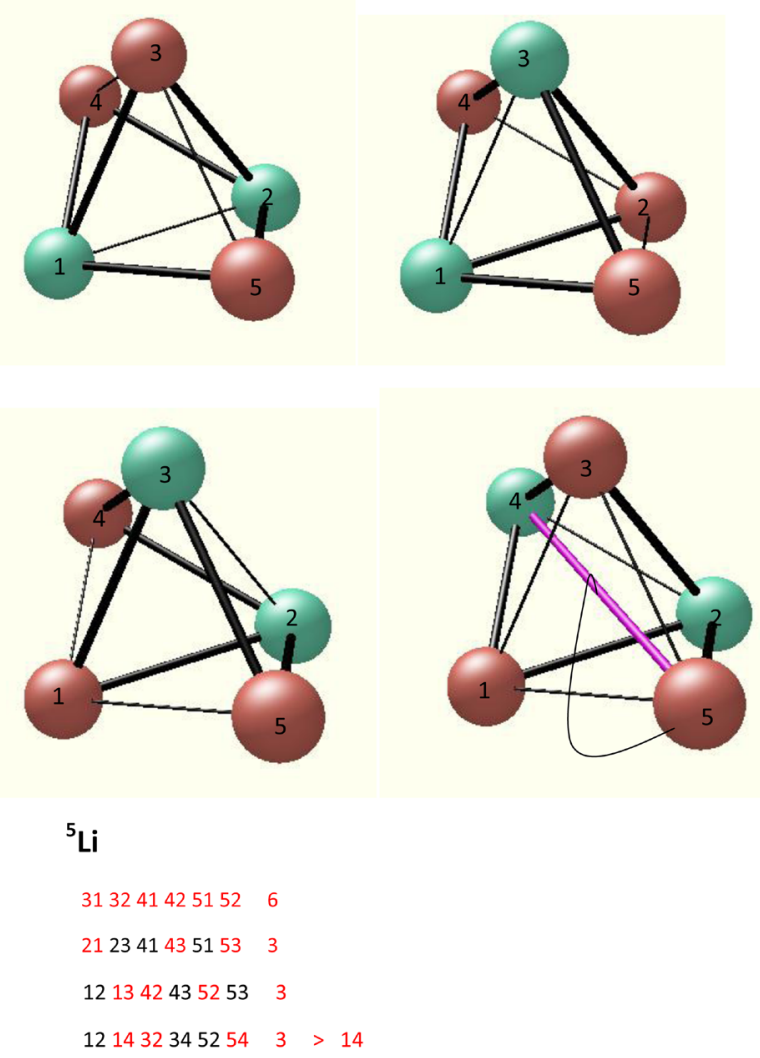

Figure 7. Number of dynamic bonds of ${ }^{5} \mathrm{Li}$. 

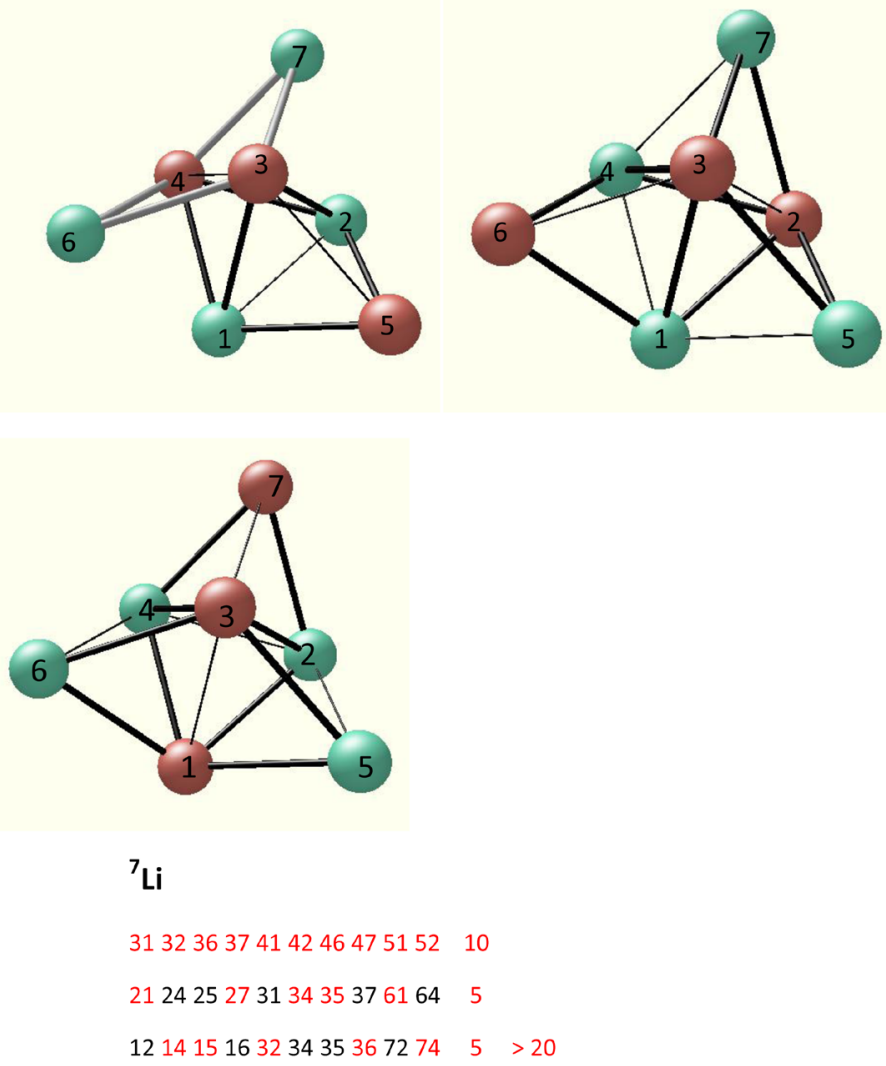

Figure 8. Number of dynamic bonds of ${ }^{7} \mathrm{Li}$.
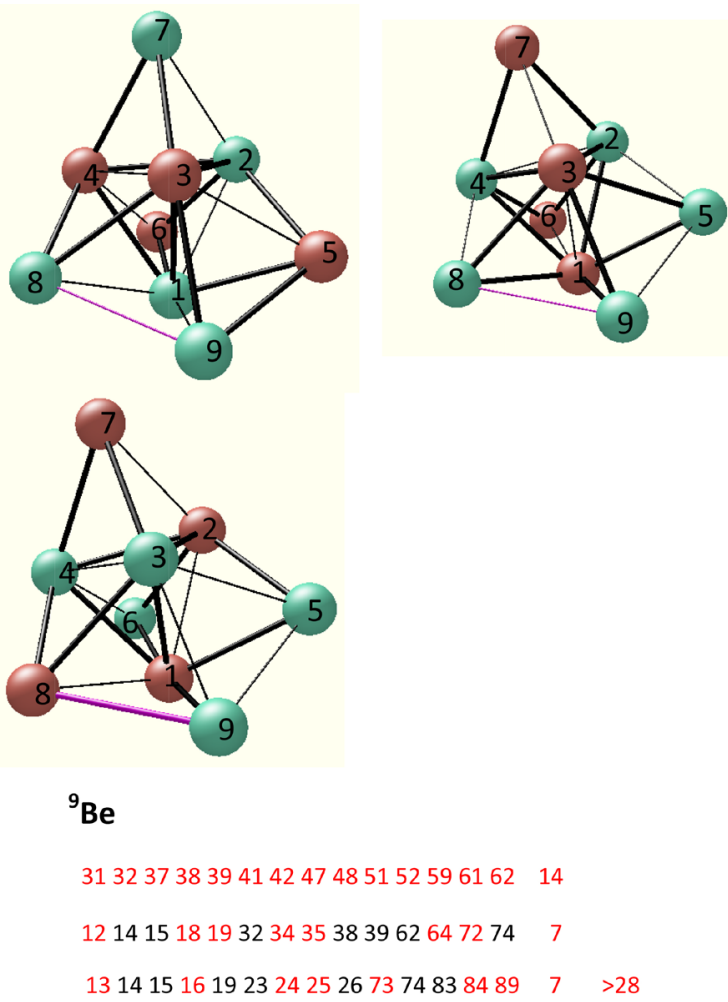

Figure 9. Number of dynamic bonds of ${ }^{9} \mathrm{Be}$. 

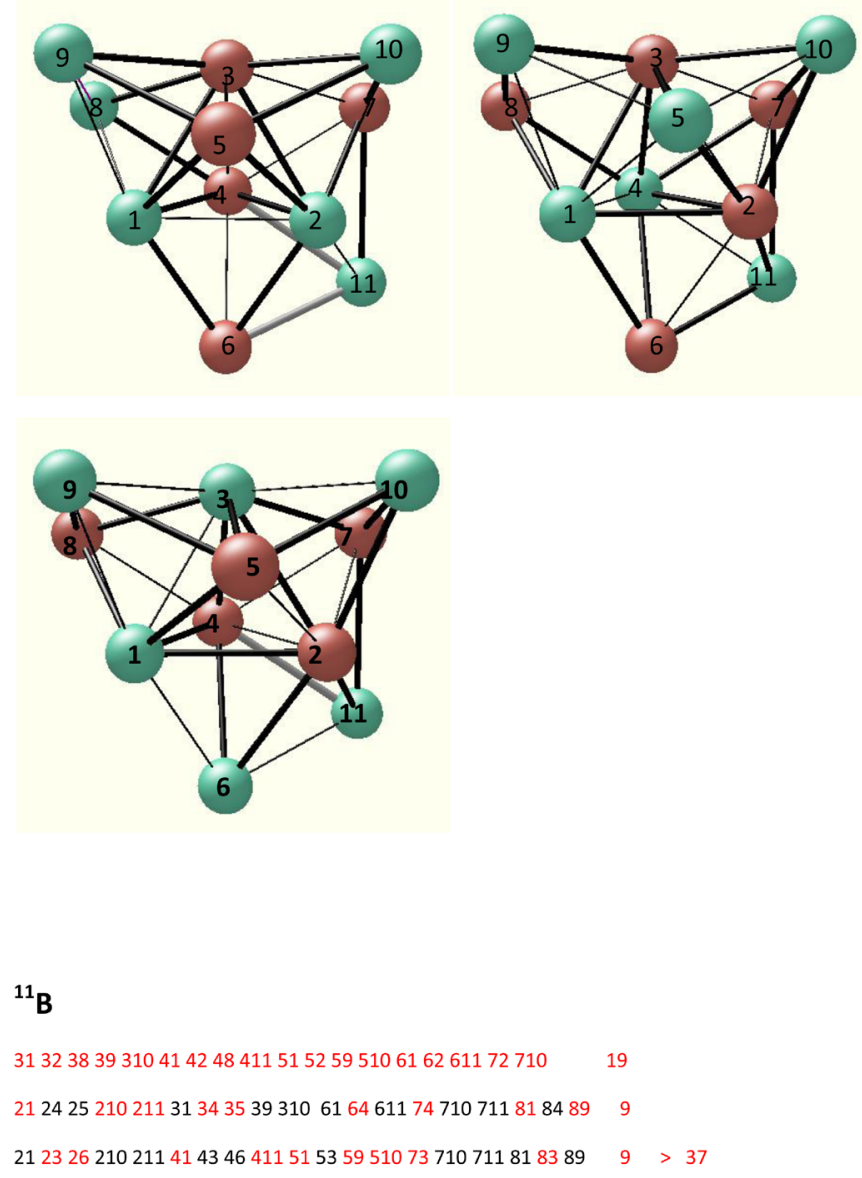

Figure 10. Number of dynamic bonds of ${ }^{11} \mathrm{~B}$.

bonds for energetic reasons and are therefore feasible but prohibited, being end points for a dynamically resonating nucleus.

To reduce the complexity of the search of resonating structures we vary the central four atoms and consequently change that of the surrounding ones following the consistency of each tetrahedron: two neutrons and two protons, each nucleon with at least two bonds.

For an additional simplification, we fix one nucleon in the central tetrahedron, for example nucleon number 3 , and vary the other three and compute the bonds with the four sequences, obtaining the first basic path.

Then we easily compute all similar three paths obtained by changing the fixed nucleon with one of the others: almost all bonds are reproduced with the exception of few new ones and, due to the central tetrahedron symmetry, the structure does not need to be changed.

We consider the mean of these new bonds over the four paths to be added to the bonds of each path as shown by the following ${ }^{12} \mathrm{C}$ example.

The sequence in Figure 11 has been obtained maintaining proton 3 fixed, changing the other nucleons 1, 2, 4 of the central tetrahedron and consequently the surrounding nucleons to follow the rules. 

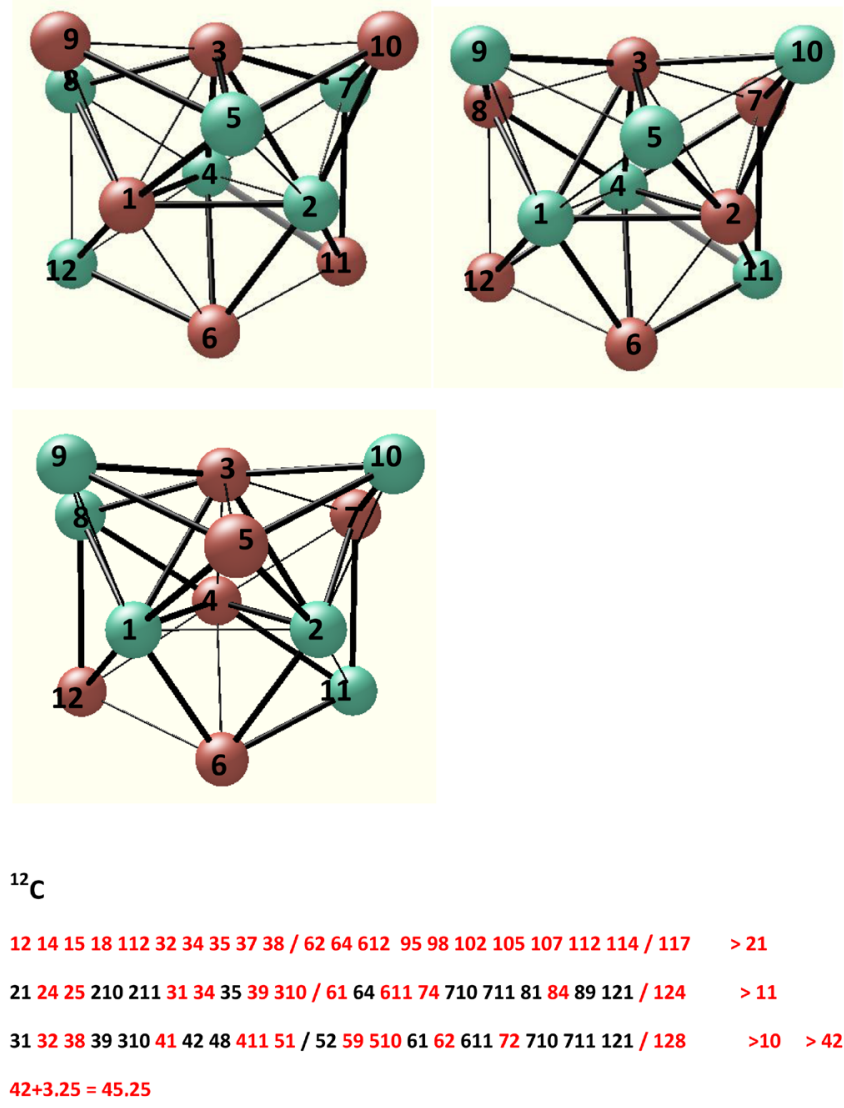

Figure 11. Number of dynamic bonds of ${ }^{12} \mathrm{C}$.

We obtain 42 bonds and to complete the count we substitute proton 3 with proton 1, 2, 4 obtaining three sequences having the same number of bonds of the first sequence for symmetry reasons.

The types of bonds are the same with the exception of about four bonds per each sequence that contribute for $13 / 4=3.25$ additional bonds for a total 45.25 bonds to be compared to 45,088 computed with Equation (1).

In Figure 12, ${ }^{14} \mathrm{~N}$ has 48 bond plus 4 that result in 52 bonds (51,202 computed with (1)) and in Figure $13{ }^{16} \mathrm{O}$ has 60 bonds plus 4 that is 64 bonds $(62,435$ computed with (1)).

We have to say that these figures can change even 2 or 3 points changing the starting structure of the sequence or changing the sequence and the criteria to estimate the residual bonds and therefore are to be considered as examples demonstrating the application of the method.

In addition from the structural analysis of bonds we have always integer numbers while from the statistical analysis over a series of sequences fractional residual bonds are obtained.

Other nuclei and isotopes have been analyzed but, for closing the show, we add the computation of dynamic bonds of ${ }^{19} \mathrm{~F}$ (Figure 14) and ${ }^{20} \mathrm{Ne}$ (Figure 15).

Table 2 reports the synthesis of the computations, most of them represented in detail in the preceding tables. 

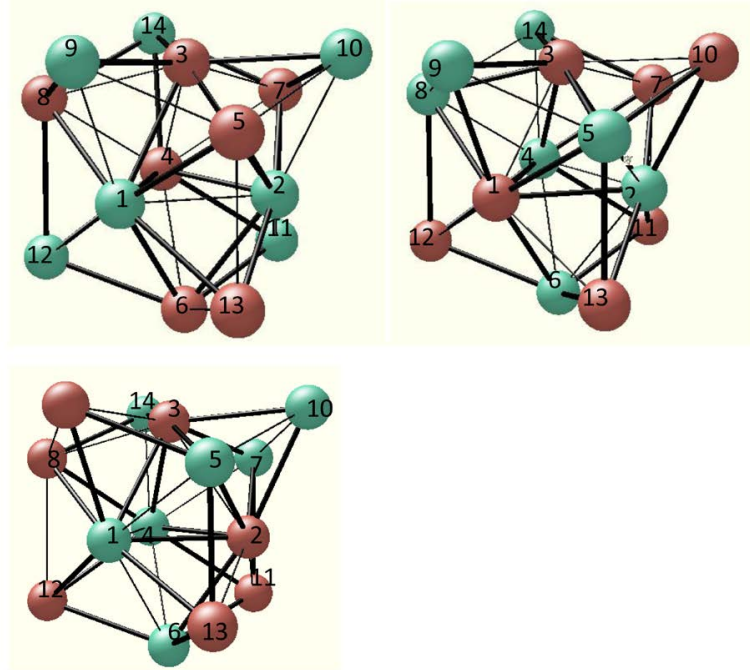

${ }^{14} \mathrm{~N}$

3132393103144142411412414 / $5152616261161272710711714 / 8189812814131132 \quad$ 26 12141516181932343538 / 393147274714102105112114116 / 124126128132135136 _1 $212425262721031343537 / 31031481848149195114116117 / 121124126131135136$ _1 >48 $48+4=52$

Figure 12. Number of dynamic bonds of ${ }^{14} \mathrm{~N}$.
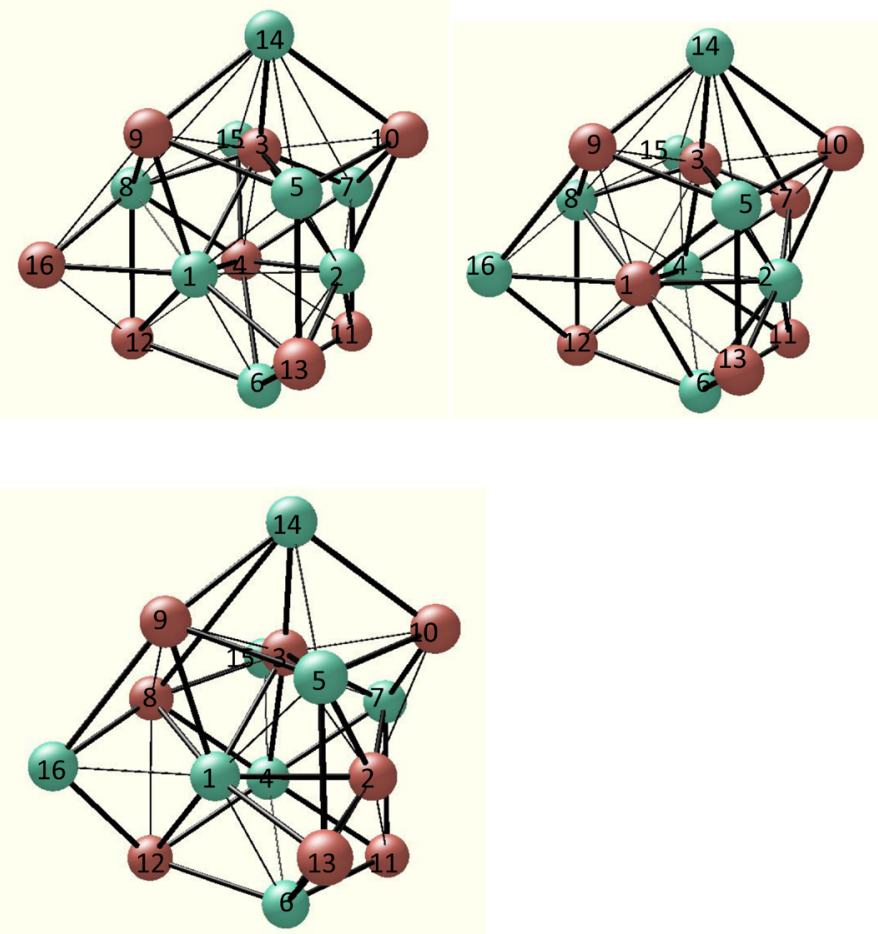

${ }^{16} \mathrm{O}$

$3132353738314315414246 / 4748415919598914102105107 / 1014112116117121126128131132135 / 136161168$ 33 $121415161811632343538 / 31431572747147159598914916 / 10210510141121141161241261281216 / 132135136 \quad 12$ $212425262731343537314 / 31581848148158169195914916 / 10510710141141161171211241261216 / 131135136 \quad 15$
$>$ > 60 $80+4=64$

Figure 13. Number of dynamic bonds of ${ }^{16} \mathrm{O}$. 

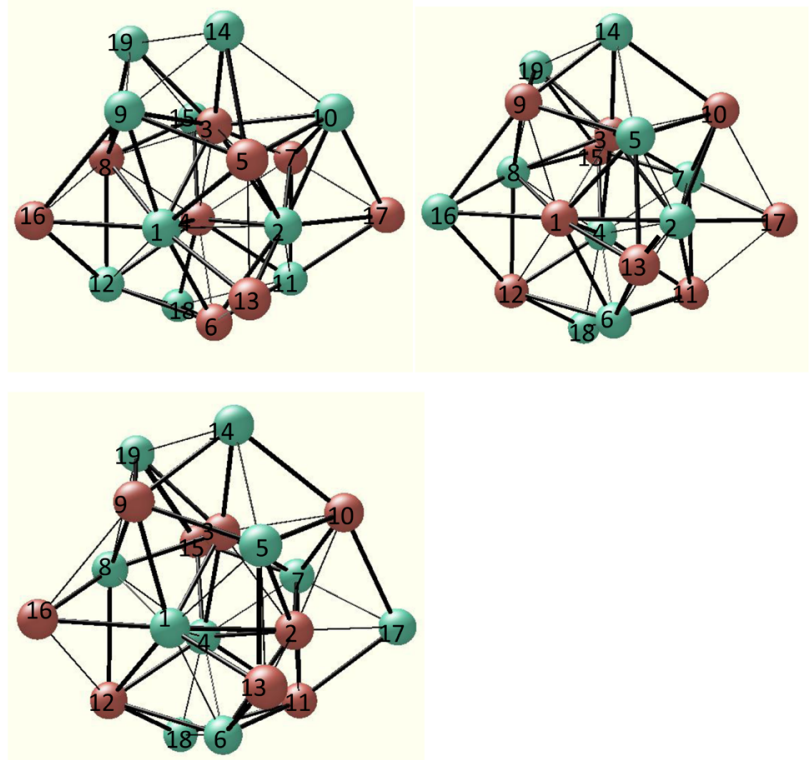

${ }^{19} \mathrm{~F}$

$3132393103143153194142411 / 4124154185152595105146162$ / 611612618727107117158189812 /815 8191311321311 $16116916121721710 / 171141$

$121415161811632343537 / 383143199598914916919102105$ / 10710141121141161171118124126128 / 12161218132135136 $1541571581519172 / 177 \quad 15$

$212425262721731343537 / 3831431991059$

$67+6=73$

Figure 14. Number of dynamic bonds of ${ }^{19} \mathrm{~F}$.
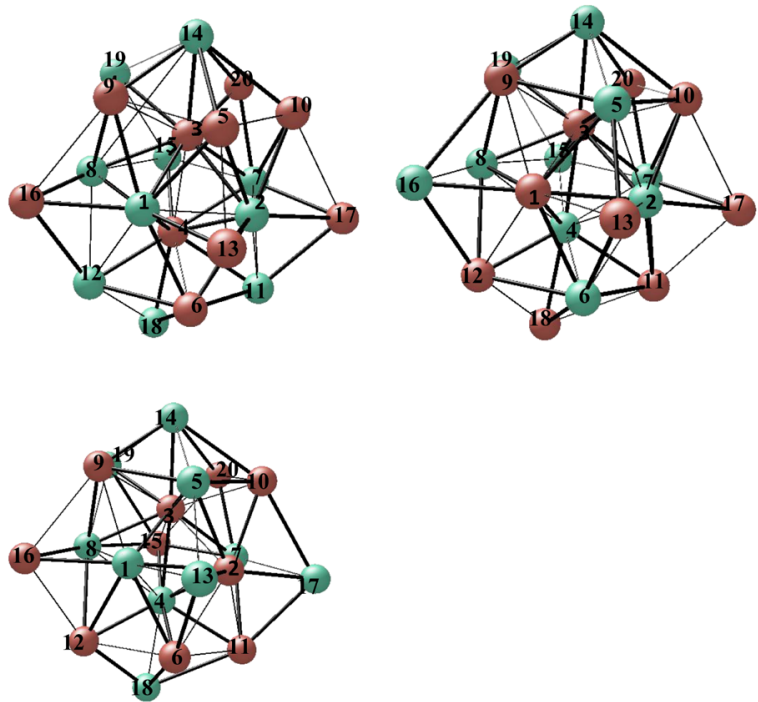

${ }^{20} \mathrm{Ne}$

(1) $4841141241541851525146162 / 61161261891989149191021071014$ / 1311321611681612 $17217717112072014 / 2015 \quad 41$

$121415161811632343537 / 38$
$177184186205207 / 2014 \quad 14$

$212425272173134353738 / 31431961646136189198914919 / 1051071014101711411611711171118121 / 1241281218154157$

$69+5.25=74.25$

Figure 15. Number of dynamic bonds of ${ }^{20} \mathrm{Ne}$. 
Table 2. Nuclear bonds from experimental and computed data.

\begin{tabular}{|c|c|c|c|c|}
\hline & N.bond (1) & N.bond (2) & N.bond (3) & N.BOND \\
\hline Nuclide & & & & Computed \\
\hline $\mathrm{H} 2$ & 1.135 & 1.518 & 1.135 & 1 \\
\hline H3 & 4.149 & 4.532 & 4.149 & 4 \\
\hline He3 & 3.776 & 4.541 & 4.172 & 4 \\
\hline $\mathrm{He} 4$ & 13.845 & 14.610 & 14.205 & 14 \\
\hline $\mathrm{He} 5$ & 13.418 & 14.184 & 13.753 & 14 \\
\hline He6 & 14.320 & 15.086 & 14.635 & 14 \\
\hline Li5 & 12.881 & 14.029 & 13.883 & 14 \\
\hline Li6 & 15.653 & 16.802 & 16.597 & 16 \\
\hline Li7 & 19.200 & 20.349 & 20.096 & 20 \\
\hline Be6 & 13.555 & 14.704 & 14.499 & 16 \\
\hline $\mathrm{Be} 7$ & 18.396 & 19.927 & 20.188 & 20 \\
\hline Be9 & 28.456 & 29.987 & 30.104 & 28 \\
\hline Be10 & 31.789 & 33.320 & 33.380 & 32 \\
\hline B10 & 31.678 & 33.592 & 34.330 & 33 \\
\hline B11 & 37.282 & 39.196 & 39.851 & 37 \\
\hline $\mathrm{C} 12$ & 45.088 & 47.385 & 48.832 & 45.25 \\
\hline $\mathrm{C} 13$ & 47.508 & 49.805 & 51.153 & 48.5 \\
\hline $\mathrm{C} 14$ & 51.509 & 53.806 & 55.065 & 51 \\
\hline N14 & 51.202 & 53.882 & 56.181 & 52 \\
\hline N15 & 56.503 & 59.182 & 61.368 & 59.25 \\
\hline 016 & 62.435 & 65.498 & 68.785 & 64 \\
\hline 017 & 64.463 & 67.525 & 70.685 & 65 \\
\hline 018 & 68.398 & 71.461 & 74.503 & 68 \\
\hline F18 & 67.206 & 70.651 & 75.055 & 69.25 \\
\hline F19 & 72.309 & 75.754 & 80.018 & 73 \\
\hline $\mathrm{Ne} 20$ & 78.595 & 82.423 & 88.068 & 74.25 \\
\hline
\end{tabular}

The experimental number of bond 1, 2 and 3 consider the mass defect following the interpretation given by Equations (1), (2) and (3) and a bond energy of $2.044 \mathrm{Mev}$.

All equations fit light nuclides and provide a solution to the mystery of their excessive binding energy, while, for heavier nuclides, the comparison with computed results appear to favor the interpretation given by Equation (1).

In particular the effect of the Coulomb energy, that is considered, in the static representation of nuclei, appears to diverge from our geometrically dynamic model under the $2.044 \mathrm{Mev}$ bond energy hypothesis. 


\section{Concluding Remarks}

Our travel started from the identification of the nuclear atom as the micro engine that moves the planets and the stars through neutrino emission and controls all chemical and physical phenomena with its electronic cloud.

The engine performs a continuous neutron-proton transformation that is the origin of dynamic neutron-proton nuclear bonds, having 2.044 Mev energy, that is the mass loss in an annihilation reaction.

Therefore, neutron and protons stick together, sharing this mass loss, like multi joint twins and justifying the excessive mass defect of Deuterium, Tritium and Helium.

The travel made demonstrates that this approach is valid also for heavier nuclides and allows building up, step by step and following specific rules, the structures of all known nuclides.

If one compares this view with the shadowed or dark image of the nucleus, ever since available, he should be proud of the result.

Nevertheless, the question marks, raised by this analysis, are soaring and, first of all, the relation of some bench-scale designed nuclear structures, to the electrons' shells and to the well-known properties of atoms and molecules.

The present study has been based on known and unexplained experimental data and we think that there is still a lot to learn from careful and unbiased observation of nature.

\section{Conflicts of Interest}

The author declares no conflicts of interest regarding the publication of this paper.

\section{References}

[1] Donati, G (2004) Il legame nucleare e il peso delneutrino. La Chimica e l'Industria, 62-66.

[2] Borsanyi, S., et al. (2015) Ab Initio Calculation of the Neutron-Proton Mass Difference. https://arxiv.org/abs/1406.4088

[3] Fermi, E. (1967) Nuclear Physics, Course at the University of Chicago. The University of Chicago Press, Chicago, IL.

[4] Donati, G (2006) L'universo invisibile (neutrino, quanti e relatività). ICPN, 92-97.

[5] Donati, G. (2006) What Gravity Is. 1-22. http://www.academia.edu/11425803/

[6] Donati, G. (2017) Rethinking the Earth in the Solar System. Journal of Applied Mathematics and Physics, 5, 631-638. https://doi.org/10.4236/jamp.2017.53054

[7] Donati, G (2018) Why the Earth Quakes. Journal of Geology \& Geophysics, 7, 342. https://doi.org/10.4172/2381-8719.1000342

[8] Donati, G (2007) Il Messaggero del campoelettromagnetico. ICPN, 80-84.

[9] Donati, G (2012) The Kinetic Theory of matter and Perpetual Motion. http://www.academia.edu/8068603

[10] Donati, G (2017) The Speed of Light in a Novel Gravitational Environment. Journal of Applied Mathematics and Physics, 5, 1938-1950. 
https://doi.org/10.4236/jamp.2017.510164

[11] Donati, G. (2019) The Last Basic Question Marks of Physics: Gravity and the Nuclear Bond. Physical Science \& Biophysics Journal, 3, 118.

[12] González-Martín, G.R. (2007) Binding Energies of the Deuteron, the Neutron and the Alpha Particle from a Theoretical Geometric Model. https://arxiv.org/abs/0712.1531

[13] González-Martín, G.R. (2008) Binding Energies of the $\alpha$ Particle and the A $=3$ Isobars from a Theoretical Geometric Model. https://arxiv.org/abs/0805.0363

[14] Abbas, S.A. and Ahmad, S. (2011) A = 3 Clustering in Nuclei. International Journal of Modern Physics E, 20, 2101-2118. https://doi.org/10.1142/S0218301311019714

[15] Rybczynski, M., et al. (2017) Signatures of $\alpha$ Clustering in Ultra-Relativistic Collisions with Light Nuclei. https://arxiv.org/abs/1711.00438

[16] Fraser, R., Yépez-Martínez, H., Hess, P.O. and Parra Rodríguez, L. (2010) Phenomenological and Semimicroscopic Clustermodels and Their Phase Transitions. Journal of Physics: Conference Series, 322, 1-9. https://doi.org/10.1088/1742-6596/322/1/012010

[17] Y’epez-Mart'inez, H., Fraser, P.R., Hess, P.O. and Lévai, G. (2012) Phenomenological and Microscopic Cluster Models. I. The Geometric Mapping. Physical Review C, 85, Article ID: 014316. https://doi.org/10.1103/PhysRevC.85.014316 https://journals.aps.org/prc/abstract/10.1103/PhysRevC.85.014316

[18] Bihari, G. (2017) Geometric Models of Atomic Nuclei. World Journal of Nuclear Science and Technology, 7, 206-222. https://doi.org/10.4236/wjnst.2017.73017 


\section{Notation}

A: mass number

$\mathrm{Z}$ : atomic number

$\mathrm{N}$ : neutron number $=A-Z$

$\mathrm{M}$ : atomic mass

$\mathrm{m}\left({ }^{1} \mathrm{H}\right)$ : mass of Hydrogen atom

$\mathrm{mp}, \mathrm{mn}$, me proton, neutron and electron mass

$\Delta \underline{\mathrm{m}}$ : mass defect

C: Coulomb energy

$n$, pneutron, proton

$\beta^{+}, \beta$ : positron, electron

$\gamma$ : annihilation radiation

$K_{1}, K_{2}, K_{3}$ : Reaction constants in Equation (5)

Fo: neutrino flux per gram of matter and second

$\mu$ : neutrino mass

rno: nucleon mass

$\mathrm{r}_{\mathrm{n}}$ : nucleon radius

c: speed of light 